\title{
Precisely Localizing Wavelength Sensitive Point-Spread Functions Engineered With a Silicon Oxide Phase Plate
}

\author{
Jason T. Martineau ${ }^{1}$, Erik M. Jorgensen ${ }^{2}$, Rajesh Menon $^{3}$, Jordan Gerton ${ }^{1}$ \\ 1. University of Utah, Department of Physics and Astronomy, Salt Lake City, USA \\ 2. University of Utah, Department of Biology, Salt Lake City, USA \\ 3. University of Utah, Department of Electrical and Computer Engineering, Salt Lake City, USA
}

Localization microscopy (LM) techniques such as PALM [1] and STORM [2] have become important tools for studying cells at nanometer length scales. Moreover high-speed sCMOS cameras have allowed LM to obtain useful data on very small time scales [3]. However, it is not currently feasible to observe the precise motions of three or more different proteins simultaneously using LM because of the broadness of the emission spectra of fluorescent tags. Fluorescent dyes that blink well tend to emit in the red or far red $(\lambda>600 \mathrm{~nm})$. In the case of three or more dyes at the red end of the spectrum, the level of cross talk is unacceptably high.

To address this problem, we have taken an approach based on point-spread function (PSF) engineering. We have developed a silicon oxide phase plate (manufactured at the University of Utah Nano-Fabrication facility) that induces a wavelength-dependent PSF when placed in the Fourier plane of a microscope. We have dubbed it the "clover" PSF because it looks like a four-leaf clover. While others have done similar experiments $[4 ; 5]$, to our knowledge we are the first to use a simple dielectric phase plate in this way. In contrast to using a liquid crystal spatial light modulator, our dielectric element is polarization insensitive. This allows us to avoid discounting the photon-budget caused by splitting and polarizing the signal. Additionally, our design is simple, which we believe will extend its utility in the future to many different areas of research.

To demonstrate the utility of the clover PSF in precisely finding an emitter's position and color, we imaged a sample (Fig. 1) prepared with three different types of $40 \mathrm{~nm}$ diameter beads (Thermo-Fisher Fluospheres: F8795, F8793, F8789). We used a Photometrics Evolve EMCCD camera for data collection with the gain turned off. These data were fit using a custom Matlab routine that used a Fast Fourier Transform algorithm to model the expected PSF given the phase plate we used. These simulations accounted for the small translational and rotational offsets from the ideal position of the phase plate inherent in its installation. By taking 100 camera frames and binning the data in time we measured the uncertainty in localization position and wavelength as a function of the number of collected photons (Fig. 2).

Our results indicate that the clover PSF is sensitive to wavelength while maintaining spatial localization precision for photon-counts that are within those of dyes used in LM. In the future, we will insert this optic into a commercial LM microscope toward the goal of obtaining multi-color super-resolution images in biological samples. Eventually we hope to do three-color live cell imaging with this technology. However, to this end the quality of our phase plate needs to be improved because its current incarnation scatters too much signal.

\section{References:}

[1] Shtengel, G. et al, Proceedings of the National Academy of Sciences 106 (2009), p. 3125. 
[2] BATES, M. et al, Science 317 (2007), p. 1749.

[3] HuAng, F. et al, Nature Methods 10 (2013), p. 653.

[4] Shechtman, Y. et al, Nature Photonics 10 (2016), p. 590.

[5] SMITH, C. et al, Optics Express 24 (2016), p. 4996.

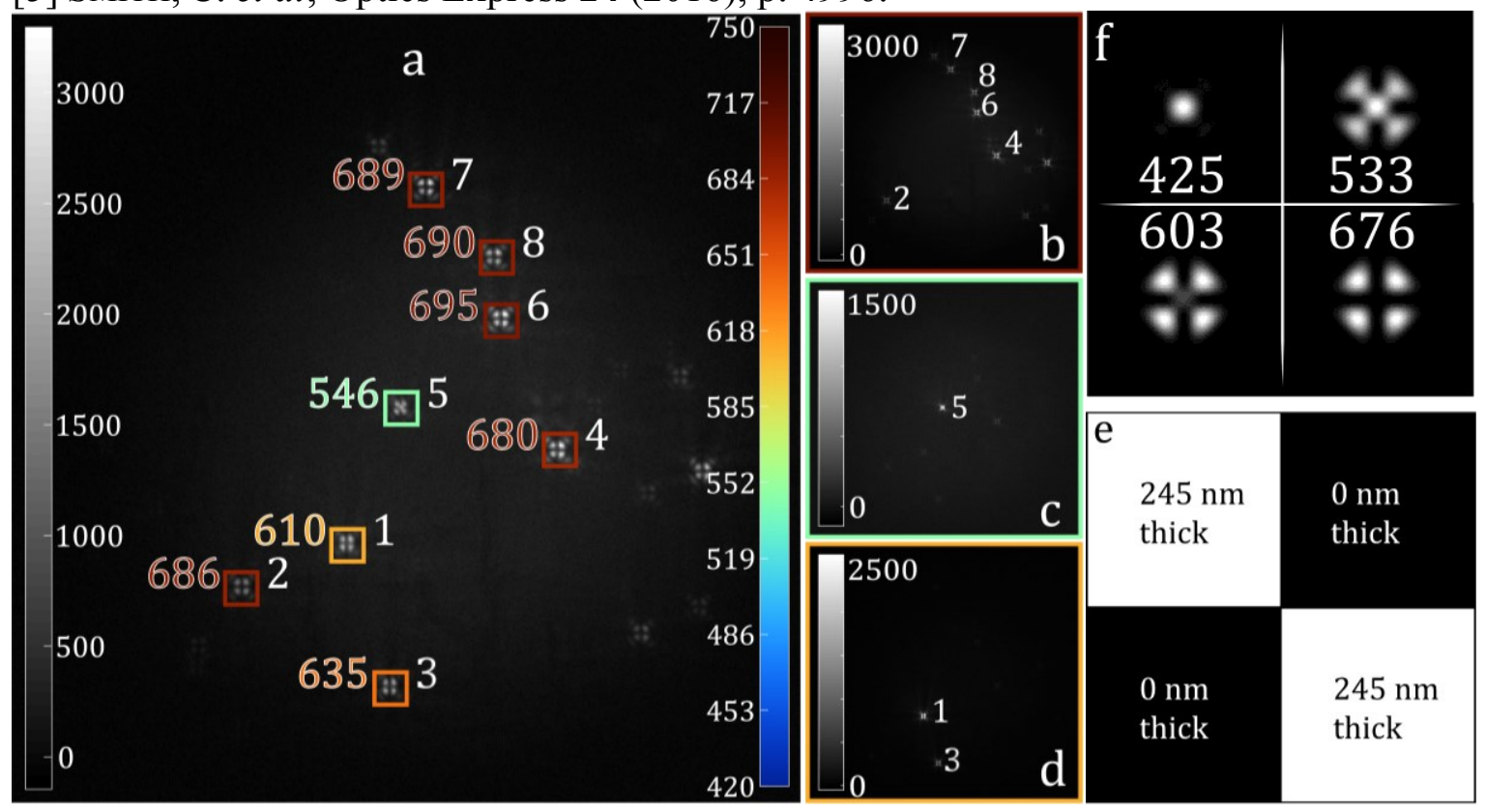

Figure 1. Panel (a) shows beads illuminated with 488, 561, and $647 \mathrm{~nm}$ lasers. The colored boxes are labeled with the wavelengths that gave the best PSF fit. Panels (b-d) show the sample illuminated only by $647,488,561 \mathrm{~nm}$ lines respectively. The gray-scale bars on the left of these panels indicate photon count. Panel (e) shows the phase plate design. Panel (f) shows simulated PSFs for four different wavelengths. All length units are nanometers.

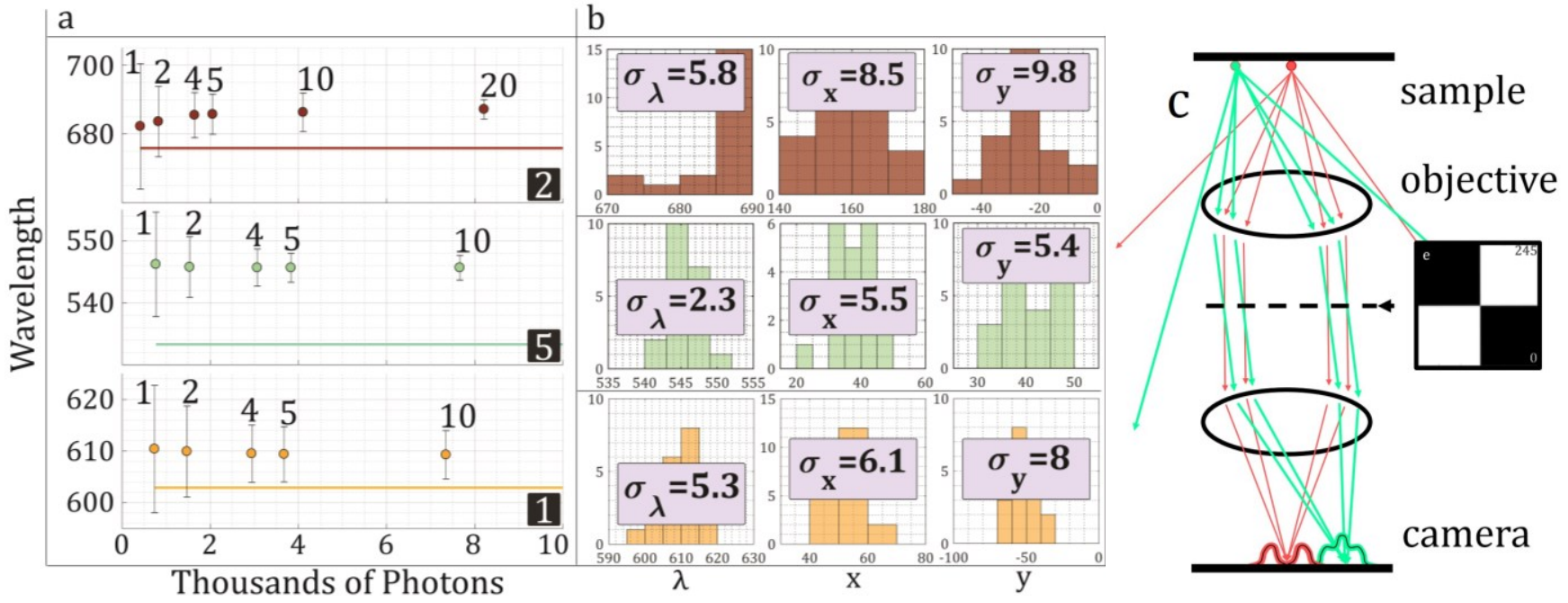

Figure 2. Each marker in (a) indicates the mean of the fit values for PSFs 2, 5, and 1 (Fig. 1). The numbers by each marker indicate the number of frames per bin for that estimation. Error bars indicate the standard deviation of these estimates. The solid lines are the theoretical spectral centers of mass for the signal. Panel (b) shows histograms for the position and wavelength estimates at five frames/bin for PSFs 2, 5, and 1. Panel (c) shows a simple diagram of the microscope. All length units are nanometers. 